\title{
The magnitue-diameter relation in galaxies
}

\author{
Sidney van den Bergh
}

\begin{abstract}
National Research Council of Canada, Dominion Astrophysical Observatory, Herzberg Institute of Astrophysics,
\end{abstract} 5071 W. Saanich Rd., Victoria, BC, V9E 2E7, Canada

Received 11 April 2008 / Accepted 21 August 2008

\begin{abstract}
Aims. We investigate the dependence of the luminosity-diameter relation of galaxies on the environment. Methods. This study is based on a comparison between the 80 galaxies in the Shapley-Ames Catalog that are located within a distance of $10 \mathrm{Mpc}$, and the luminosity-diameter relation for galaxies in great clusters such as Virgo and Coma.

Results. A relatively tight linear correlation is observed between the absolute magnitudes and the logarithms of the linear diameters of galaxies located within $10 \mathrm{Mpc}$. Surprisingly this observed power-law relationship appears to be almost independent of environment and local mass density as defined by Karachentsev \& Malakov. However, at a given luminosity, early-type galaxies are (on average) slightly more compact than are objects of a later type. Unexpectedly the present results appear to indicate that the luminosity-diameter relation for the galaxies within $10 \mathrm{Mpc}$ is indistinguishable from what is observed in the much denser Virgo cluster.

Conclusions. Galaxies appear to form an almost one-dimensional family in parameter space. It remains a mystery why the luminositydiameter relation for galaxies is so insensitive to environment.
\end{abstract}

Key words. galaxies: luminosity function, mass function

\section{Introduction}

Giuricin et al. (1988) first drew attention to the surprising fact that the luminosity-diameter relation provides the tightest of all correlations observed among the photometric parameters that can be used to characterize galaxies. Subsequently this conclusion was strengthened and confirmed by Gavazzi et al. (1996) who found a strong correlation between diameter and blue-light luminosity of galaxies, and an even tighter correlation between $H$-band luminosity and disk galaxy diameter. Similarly tight relationships can be obtained by comparing photometric and dynamical parameters describing galaxies (Faber \& Jackson 1976; Tully \& Fisher 1977). According to present ideas the formation of galaxies is a very messy and chaotic process involving both multiple mergers and complicated feed-back effects. Furthermore the history of the rate of star formation in individual galaxies can differ in a bewildering variety of ways. From currently fashionable ideas on galaxy formation one would expect environment, initial mass, angula momentum, central concentration, star formation history and merger history to all affect the evolution of disk galaxies (Dalcanton et al. 1997; Ma et al. 1998). This raises the question why parameters, such as galaxy luminosity and diameter, should still be so closely correlated at the end of widely differing evolutionary tracks in different environments. Possibly some hints about the origin of the observed power-law relation between the luminosity and the diameter of galaxies might be obtained by searching for variations of the luminosity-diameter relation as a function of galaxy environment, which may itself be characterized in a variety of differing ways. Girardi et al. (1991) have pointed out that "The tightness of this relationship suggests that possible environmental effects should be detectable from the analysis of its shape in different samples". Giuricin et al. (1988) claim to have detected "appreciable differences in the galaxy-luminosity relationships for different clusters". On the other hand Girardi et al. (1991) reach the opposite conclusion and find no significant differences between the luminosity-diameter relations in a variety of environments. Similarly Gavazzi et al. (1996) obtain broadly similar luminosity-diameterrelations in differing environments. It is the purpose of the present study to re-investigate this question by comparing the luminosity-diameter relations in three particularly high quality data sets: (1) the Shapley-Ames galaxies with $D<10 \mathrm{Mpc}$, (2) the disk galaxies in the Virgo cluster, and (3) a number of other more distant Abell clusters.

\section{Database}

Physical information is most complete for the nearest and the brightest galaxies. Such galaxies therefore provide an excellent laboratory for study of the relationship between the luminosities and diameters of galaxies. A catalog of 451 galaxies, that are believed to be closer than $10 \mathrm{Mpc}$, has recently been published by Karachentsev et al. (2004). A listing of the brightest galaxies (which is complete to $B \sim 13$ ) was given by Shapley \& Ames (1932) and has been updated and revised by Sandage \& Tammann (1981). The Revised Shapley-Ames Catalog, which contains 1276 galaxies, is particularly valuable because it provides complete and homogeneous information on the morphologies of all of the brightest galaxies based on uniform classifications that were made by expert morphologists on the basis of inspection of plates that were almost all obtained with large reflecting telescopes. The Karachentsev et al. and Revised Shapley-Ames catalogs have 79 galaxies in common, or 80 if the Milky Way System (which was omitted from the Shapley-Ames Catalog) is included. A listing of all of these galaxies is given in Table 1. For each object this table lists: (1) the galaxy name, (2) the major axis linear diameter $A_{25}$ taken 
Table 1. Data on all Shapley-Ames galaxies within $10 \mathrm{Mpc}$.

\begin{tabular}{|c|c|c|c|c|c|}
\hline " Name & $\overline{\overline{A_{25}}}$ & $\overline{\overline{M_{B}}}$ & $\overline{\overline{\theta \theta}}$ & Type & $\overline{\mathrm{Gr}}$ \\
\hline N 55 & 12.05 & -18.06 & -0.4 & $\mathrm{Sc}$ & \\
\hline N 147 & 3.16 & -14.79 & +3.0 & $\mathrm{dE} 5$ & G \\
\hline N 185 & 2.51 & -14.76 & +2.3 & $\mathrm{dE} 3$ & $\mathrm{G}$ \\
\hline N 205 & 4.49 & -16.15 & +3.7 & $\mathrm{~S} 0 / \mathrm{E} 5$ & G \\
\hline N 221 & 1.99 & -15.96 & +6.8 & E2 & $\mathrm{G}$ \\
\hline N 224 & 35.87 & -21.58 & +4.6 & Sb I-II & $\mathrm{G}$ \\
\hline N 247 & 20.66 & -18.81 & +1.3 & Sc III-IV & $\mathrm{G}$ \\
\hline N 253 & 22.98 & -21.37 & +0.3 & $\mathrm{Sc}$ & G \\
\hline SMC & 5.28 & -16.35 & +3.5 & Im IV-V & $\ldots$ \\
\hline N 300 & 12.95 & -17.92 & -0.3 & $\mathrm{Sc}$ III & \\
\hline N 404 & 3.25 & -16.49 & -1.0 & So & G \\
\hline N 598 & 16.09 & -18.87 & +2.0 & $\mathrm{Sc}$ II-III & $\mathrm{G}$ \\
\hline N 625 & 5.96 & -16.53 & -0.4 & Amorph & $\ldots$ \\
\hline N 628 & 22.54 & -19.84 & 0.0 & $\mathrm{Sc} \mathrm{I}$ & F \\
\hline N 672 & 13.19 & -18.76 & +3.8 & SBc III & $\mathrm{F}$ \\
\hline N 891 & 30.80 & -20.59 & -1.2 & $\mathrm{Sb}$ & $\mathrm{F}$ \\
\hline N 925 & 28.60 & -19.88 & -0.9 & SBc II-III & $\mathrm{F}$ \\
\hline N1313 & 11.45 & -19.02 & -1.6 & SBc III-IV & $\cdots$ \\
\hline N1569 & 3.34 & -18.17 & -0.4 & Sm IV & $\mathrm{F}$ \\
\hline N1705 & 2.67 & -15.86 & -1.7 & Amorph & \\
\hline LMC & 9.75 & -17.93 & +3.6 & SBm III & G \\
\hline N2366 & 5.71 & -16.02 & +1.0 & SBm IV-V & G \\
\hline N2403 & 19.43 & -19.29 & 0.0 & Sc III & G \\
\hline N2537 & 3.49 & -16.65 & -1.4 & $\mathrm{Sc}$ III & $\mathrm{F}$ \\
\hline N2683 & 16.18 & -20.43 & +0.2 & $\mathrm{Sb}$ & $\mathrm{F}$ \\
\hline N2784 & 15.86 & -19.72 & +2.0 & So & $\mathrm{F}$ \\
\hline N2787 & 7.13 & -18.50 & -1.9 & $\mathrm{SB} 0 / \mathrm{a}$ & $\mathrm{F}$ \\
\hline N2903 & 28.94 & -21.00 & +1.8 & Sc I-II & $\mathrm{F}$ \\
\hline N2976 & 5.57 & -17.10 & +2.7 & Sd III-IV & G \\
\hline N3031 & 26.85 & -21.06 & +2.2 & Sb I-II & $\mathrm{G}$ \\
\hline N3034 & 10.93 & -19.63 & +2.7 & Amorph & $\mathrm{G}$ \\
\hline N3109 & 5.81 & -15.68 & -0.1 & Sm IV & $\mathrm{F}$ \\
\hline N3077 & 6.14 & -17.76 & +1.9 & Amorph & G \\
\hline N3115 & 17.13 & -20.82 & +1.9 & So & $\mathrm{F}$ \\
\hline N3274 & 3.50 & -16.16 & -0.3 & S IV & G \\
\hline N3344 & 14.16 & -19.03 & -1.5 & SBbc I & $\mathrm{F}$ \\
\hline N3351 & 20.53 & -19.88 & +0.8 & $\mathrm{SBb}$ II & $\mathrm{C}$ \\
\hline N3368 & 21.82 & -20.42 & +0.6 & Sab II & $\mathrm{C}$ \\
\hline N3377 & 15.27 & -19.10 & +0.8 & E6 & $\mathrm{C}$ \\
\hline N3379 & 17.46 & -20.10 & +1.0 & E0 & $\mathrm{C}$ \\
\hline N3384 & 15.91 & -19.55 & +1.2 & SB0 & $\mathrm{C}$ \\
\hline N3412 & 9.94 & -18.76 & +1.9 & SB0 & $\mathrm{C}$ \\
\hline N3489 & 9.60 & -19.45 & +1.5 & $\mathrm{~S} 0 / \mathrm{Sa}$ & $\mathrm{C}$ \\
\hline N3593 & 9.00 & -17.77 & -1.7 & $\mathrm{Sa}$ & $\mathrm{G}$ \\
\hline N3621 & 22.05 & -19.81 & -1.9 & $\mathrm{Sc}$ III & $\ldots$ \\
\hline N3627 & 23.98 & -21.14 & -0.7 & $\mathrm{Sb}$ II & G \\
\hline N3738 & 3.52 & -16.61 & -1.0 & Sd III & $\mathrm{C}$ \\
\hline N4144 & 12.89 & -18.25 & -0.9 & Scd III & $\mathrm{C}$ \\
\hline N4190 & 1.73 & -14.33 & 0.0 & Sm IV & $\mathrm{C}$ \\
\hline N4214 & 7.05 & -17.19 & -0.7 & SBm III & $\mathrm{C}$ \\
\hline N4236 & 23.58 & -18.59 & -0.4 & SBd IV & $\mathrm{G}$ \\
\hline N4244 & 16.60 & -18.60 & 0.0 & Scd & $\mathrm{C}$ \\
\hline N4258 & 35.61 & -21.25 & -0.7 & $\mathrm{Sb}$ II & $\mathrm{C}$ \\
\hline N4395 & 17.30 & -17.85 & +0.1 & Sd III-IV & $\mathrm{C}$ \\
\hline N4449 & 7.21 & -18.27 & 0.0 & Sm IV & $\mathrm{C}$ \\
\hline N4460 & 8.92 & -17.89 & -0.7 & $\mathrm{Sbc}$ & $\mathrm{C}$ \\
\hline N4594 & 21.10 & -21.90 & +0.3 & Sab & $\mathrm{C}$ \\
\hline N4605 & 7.41 & -17.96 & -1.1 & $\mathrm{Sc}$ III & $\mathrm{G}$ \\
\hline N4736 & 14.80 & -19.83 & -0.5 & $\mathrm{Sab}$ & $\mathrm{C}$ \\
\hline N4826 & 20.41 & -20.90 & -1.7 & Sab II & $\mathrm{F}$ \\
\hline N4945 & 17.41 & -20.51 & +0.7 & $\mathrm{Sc}$ & \\
\hline N5068 & 14.76 & -19.04 & -1.4 & SBc II-III & G \\
\hline N5102 & 7.20 & -18.08 & +0.7 & So & $\ldots$ \\
\hline N5128 & 28.88 & -20.77 & +0.6 & $\mathrm{~S} 0+\mathrm{Spec}$ & \\
\hline N5204 & 6.17 & -16.75 & -1.1 & Sd IV & $\mathrm{C}$ \\
\hline
\end{tabular}

Table 1. continued.

\begin{tabular}{lrrrll}
\hline \hline Name & $A_{25}$ & $M_{B}$ & $\theta$ & Type & Gr \\
\hline N5194 & 24.44 & -21.34 & +4.1 & Sbc I-II & G \\
N5195 & 11.83 & -19.22 & +5.0 & SB0 & C \\
N5236 & 17.37 & -20.43 & +0.8 & SBc II & $\ldots$ \\
N5253 & 5.04 & -17.38 & +0.5 & Amorph & $\ldots$ \\
N5457 & 61.44 & -21.23 & +0.6 & Sc I & C \\
N5474 & 9.95 & -17.74 & +2.0 & Scd IV & C \\
N5585 & 8.92 & -17.82 & -0.8 & Sd IV & C \\
Milky Way & $25.0:$ & -20.8 & +2.5 & Sbc: & G \\
I4662 & 1.55 & -15.13 & -0.9 & Im III & $\ldots$ \\
N6503 & 9.03 & -18.08 & -1.2 & Sc III & F \\
N6822 & 2.71 & -15.22 & +0.6 & Im IV-V & G \\
N6946 & 25.96 & -20.86 & +0.7 & Sc II & F \\
I5052 & 7.64 & -18.23 & -2.2 & Sd & $\ldots$ \\
I5152 & 2.91 & -15.67 & -1.1 & Sdm IV-V & $\ldots$ \\
N7793 & 9.96 & -18.53 & +0.1 & Sd IV & $\ldots$ \\
\hline
\end{tabular}

from the compilation of Karachentsev et al. (2004). This diameter was corrected for galaxy inclination and Galactic extinction in the manner of the RC2 Catalog (de Vaucouleurs et al. 1976). The value of $A_{25}$ would (after scaling to a common distance scale) be essentially the same as the diameter $D_{0}$ of De Vaucouleurs et al. and twice the value $R$ used by Gavazzi et al. (1996). In their catalog Karachentseve et al. (2004) indicate which method was used to derive the distance of each individual galaxy within a global framework in which a Hubble parameter of $72 \mathrm{~km} \mathrm{~s}^{-1} \mathrm{Mpc}^{-1}$ was adopted, (3) the integrated magnituds blue $M_{B}$ was taken from Karachentsev et al. (2004), (4) the adopted tidal index $\theta$ is based on the local mass density (Karachentsev \& Malakov 1999). In this relation the zeropoint has been set in such a way that $\theta=0$ when the Keplerian cyclical period of a galaxy, with respect to its main disturber, equals the cosmic Hubble time $1 / H_{\circ}$. Galaxies with $\theta<0$ may be considered as undisturbed (isolated) objects. A caviat is, of course, that the real orbital periods will be affected by the unknown distribution of dark matter. (5) The adopted morphological types are slightly simplified versions of the classifications given in Sandage \& Tammann (1981). Finally, (6) galaxies north of $\delta=-27^{\circ}$ (which are visible on the Palomar Sky Survey) were, on the basis of visual inspection, assigned to clusters (C), groups (G) or the field (F) environments (van den Bergh 2007) plus unpublished data. Galaxies were assigned to groups if they appeared to have between three and five non-dwarf companions, or to clusters if they had six or more non-dwarf companions. The utility of this classification is attested to by the strong correlation that is observed between the $\mathrm{C}, \mathrm{G}$ and $\mathrm{F}$ assignments and the intrinsic $U-B$ colors of galaxies (van den Bergh 2007).

It might be argued that a volume-limited galaxy sample, that includes many dwarf galaxies and reaches out to a fainter limiting isophote, would be physically more meaningful than the present sample of Shapley-Ames galaxies within $10 \mathrm{Mpc}$. However, a distinct disadvantage of such a sample is that it would include many objects for which the photometric and morphological data are incomplete, or of low quality. Finally many dwarf galaxies would be excluded from the sample because their surface brightness never attains the $25 \mathrm{mag} \operatorname{arcsec}^{-2}$ surface brightmass that defines the $A_{25}$ diameter.

The fact that the Shapley-Ames sample of nearby galaxies, and the Binggeli et al. (1984) sample of Virgo galaxies, are both luminosity-limited facilities a comparison between 

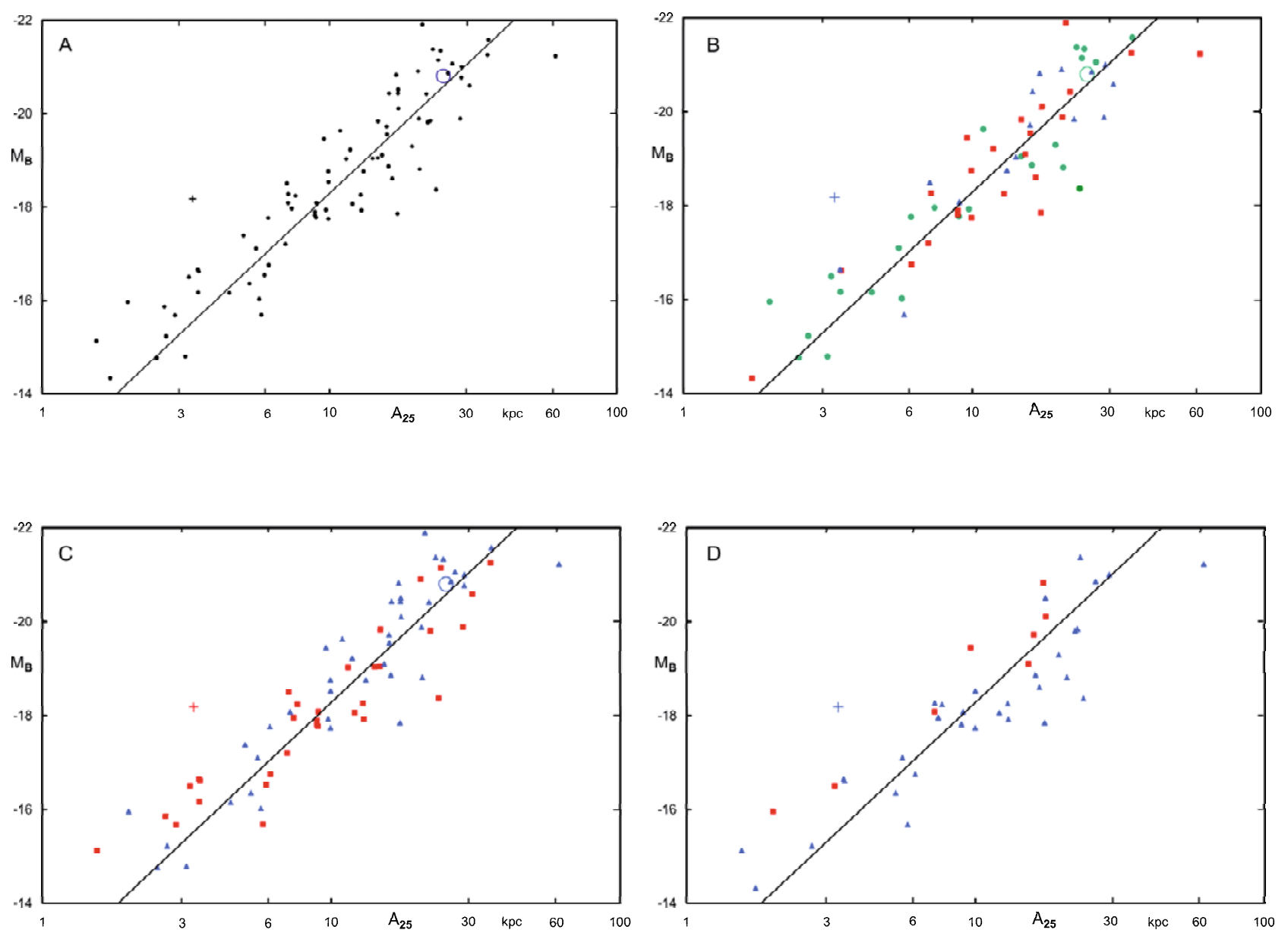

Fig. 1. This plot shows the relationship between the luminosities and the diameters of Shapley-Ames galaxies within $10 \mathrm{Mpc}$. Also shown as a solid line is the luminosity-diameter relation for the Virgo cluster according to Girardi et al. (1991). Figure 1A shows that nearby galaxies and those in the Virgo cluster follow the same power-law relationship. The deviant galaxy NGC 1569 is plotted as a plus sign. The Milky Way system is shown as a small circle. Figure 1B shows the Luminosity-Diameter relation for galaxies in clusters (red squares), in groups (green circles) and in the field (blue triangles). The Milky Way System is shown as a green circle. The figure shows a surprising absence of dependence of position in this diagram on environment. Figure 1C shows the Luminosity-Diameter relation for galaxies with $\theta<0$, which may be considered as undisturbed isolated objects (red squares). Galaxies with $\theta>0$, are plotted as blue triangles. These objects have Keplerian cyclical periods smaller than the Hubble time $1 / H_{0}$. The small blue circle represents the Milky Way System. The plot shows no evidence for a clear-cut relationship between the Karachentseve \& Malakov tidal index and deviations from the mean luminosity-diameter relation. Finally Fig. 1D shows the luminosity-diameter relation for early-type (E+S0+S0/Sa) galaxies (red squares) and late type (Sc, Scd, Sd, Sm, Im) galaxies (blue triangles). Not unexpectedly this figure shows that at, a given luminosity, early-type galaxies are (on average) slightly more compact than are objects of later morphological types.

the luminosity-density relations in these two very different environments. By the same token the data compiled by Gavazzi et al. are subsamples of the Zwicky et al. (1961-1968) cata$\log$, which comprises luminosity selected galaxies that that have $B \leq 15.7$, allowing one to compare galaxies in some Abell clusters with comparable data for field galaxies in the "Great Wall".

Yet another approach might have been to examine the relation between the luminosities of galaxies and their half-light radii. However, this has the disadvantage that the $M_{B}$ versus $\log r_{e}$ relation is distinctly non-linear (Binggeli et al. 1984), whereas (over a range of many hundreds in luminosity) the relation between $A_{25}$ diameter and galaxy luminosity is a simple power law. Finally one might argue that it would have been better to use the infrared, rather than the blue, luminosities of galaxies. Such $I$-band magnitudes are expected to exhibit a closer correlation with galaxy mass than is the case for $B$-band magnitudes. However, any dependence on environmental factors is more likely to show up in the $B$ images which are much more strongly affected by star formation and dust.

\section{Discussion}

Figure 1A shows a plot of galaxy diameter $A_{25}$ in kpc versus galaxy absolute magnitude $M_{B}$ for all of the galaxies listed in Table 1. This figure shows that, over a remarkably large range in luminosity, galaxies exhibit a reasonably tight power-law relation between luminosities and diameter. This result is both unexpected and puzzling. One might have expected the chaotic merger history of galaxies, and their widely differing histories of star formation, to have produced a broad spectrum of size-luminosity relationships. Furthermore the sizes of some galaxies are likely to have been affected by feedback produced by active galactic nuclei during early phases of their evolution. Figure 1 shows that, after reducing the Girardi et al. (1991) observations to the distance scale used in the present paper, the objects in Table 1 are well-represented by the relation

$$
\begin{gathered}
M_{B}=-12.65-5.7 \log A_{25}, \\
\pm 01 \quad \pm 0.2
\end{gathered}
$$


that Girardi et al. (1991) derived for 177 disk galaxies in the Virgo cluster. It should be emphasized that the errors quoted in Eq. (1) are probably underestimated because they do not include systematic effects that might result from the bias that is introduced by incompleteness at the faint end of the galaxy sample that these authors used.

The rms dispersion of the galaxies listed in Table 1 derived from the regression line defined by Eq. (1) is 0.8 mag. From a similar study of 533 disk galaxies in eight relatively nearby rich clusters and in the inter-cluster "Great Wall" Gavazzi et al. (1996) find a slope of 6.6, which is marginally greater than the value of $5.7 \pm 0.2$ that Girardi et al. (1991) found for the Virgo cluster. Gavazzi et al. argue that the power-law relationship between the radii and the masses of galaxies suggest "that to the first order the process of galaxy evolution can be described with a single parameter; the initial mass". It may be challenging to reconcile this conclusion with the hierarchical merging scenario in which the initial mass concept appears meaningless. In a different context Woo et al. (2008) have recently found that the Local Group dwarfs basically define a one-parameter "fundamental line" primarily driven by steller mass.

In Fig. 1 distance errors will cause a galaxy to slide along a line with slope -5.0 (corresponding to constant surface brightness), which is close to the observed slope of -5.7 in Eq. (1). As a result even moderately large errors in distance estimates for individual galaxies will not add significantly to the observed dispersion of galaxies around the line defined by Eq. (1). The most strongly deviant object (marked by a plus sign) is NGC 1569 (see panel \#336 of Sandage \& Bedke 1994), which is unusually small for its luminosity. Van den Bergh (1960) classifies it as Ir pec III-IV? It appears likely that this object is too bright for its diameter because it underwent a violent star-burst in the relatively recent past (Greggio et al. 1998). It is noted in passing that Grocholski et al. (2008) have recently used HST observations to show that NGC 1569 is more distant than previously believed and it probably a member of the IC 342 group. From the data in Table 1 it is also seen that NGC 221 (M32) is smaller than would be predicted by Eq. (1). The reason for this is, no doubt (Faber 1973), that M32 has been stripped of its outer envelope by one or more strong tidal encounters with M31.

The largest galaxy within a sphere of radius $10 \mathrm{Mpc}$ is NGC 5457 (M101). This object is almost twice as large as the two next largest galaxies with $D<10 \mathrm{Mpc}$ : the Andromeda galaxy and NGC 4258 (M 106). Peebles (2007) has drawn attention to the fact that M101 is located near the edge of the Local Void, rather than in the dense central region of the Local Super-cluster.

Figure 1B shows that field $(F)$, group $(G)$ and cluster $(C)$ galaxies are all distributed similarly relative to the line defined by Eq. (1). This result is slightly puzzling because van den Bergh (2007) found a very strong correlation between the morphological types of galaxies and their assignment to $F, G$ and $C$ environments. One might perhaps have expected cluster members (which are mainly of early morphological types) to be smaller than field galaxies that mostly have late morphological types.

Figure $1 \mathrm{C}$ shows that field galaxies, and objects that are bound on timescales $<1 / H_{\circ}$, are distributed in the same fashion. Taken at face value these results suggest that the position of an object on the luminosity-diameter relation is essentially independent of the environment in which it finds itself. This result is again somewhat counterintuitive because one might have expected compact early-type galaxies to occur predominantly among objects that are bound on a Hubble time, whereas more extended late-type galaxies would perhaps have been expected to predominate among unbound field galaxies.
Finally Fig. 1D shows that early-type and late-type galaxies are displaced relative to each other. Not unexpectedly the figure shows that, at a given luminosity, early-type galaxies are more compact than galaxies of late type. In other words, the luminosity-diameter relation for early-type galaxies is slightly displaced towards smaller diameters relative to that for late- type galaxies. These small deviations show that galaxies are almost, but not exactly, a one parameter family. In this connection it is of interest to note that Gavazzi et al. (1996) find that the power law relationship between galaxy luminosity and diameter is even tighter in $H$-band than it is in the $I$-band. This is no doubt due to the fact that environmental factors, that may influence galaxy formation and the presence of dust will, affect the blue luminosity of a galaxy more that they will its infra-red luminosity.

One of the main result of the present investigation is that the relatively well-studied Virgo cluster exhibits a luminosity- diameter relationship that is indistinguishable from that of the lower density region containing the Shapley-Ames galaxies that are situated within a distance of $10 \mathrm{Mpc}$. In their study of the Virgo cluster Girardi et al. (1991) divided their Virgo sample into three sub-regions: an inner shell within $R \leq 0.5 \mathrm{Mpc}$ of the center of the Virgo cluster, an intermediate shell with $0.5<R \leq 1.0 \mathrm{Mpc}$, and an outer shell with $R>1.0 \mathrm{Mpc}$. These authors found that these three subsamples exhibited extremely similar luminositydiameter relations. More fragmentary data that Girardi et al. collected on the diameters of other (mainly more distant) clusters do not appear to show clear-cut differences between the luminositydiameter relation for the Virgo cluster and those for other dense clusters.

Girardi et al. (1991) have also compared the luminositydiameter relations of objects in Tully's (1988) Nearby Galaxies Catalog, which lists galaxies out to a redshift of $3000 \mathrm{~km} \mathrm{~s}^{-1}$ corresponding to $R \simeq 42 \mathrm{Mpc}$. Within this largere volume these authors find no significant differences between the luminositydiameter relations for galaxies that Tully assigns to (1) the field, (2) groups, and (3) clusters. Girardi et al. therefore conclude that "These results showed that any possible environmental effect is not strong enough to affect significantly the L-D relation for disk galaxies in the samples used in the present paper". The results obtained from Tully's catalog are therefore entirely consistent with those shown in Fig. 1B which exhibits a similar independence from environmental effects for galaxies that van den Bergh (2007) assigned to clusters, groups and the field from visual inspection of the prints of the Palomar Sky Survey. Furthermore these results are also consistent with the data plotted in Fig. 1C, which show no obvious dependence of the luminosity-diameter relation among nearby galaxies on the $\theta$ index of Karachentsev \& Malakov (1999). Finally Gavazzi et al. (1996) find no systematic difference between the luminosity-diameter relations in dense Abell clusters and among the more isolated individual galaxies in the "Great Wall". The fact that Gavazzi et al. find a much tighter correlation between $H$-band luminosity and radius than between $B$-band luminosity and galxy radius confirms the suspicion that environmental effects are smaller at long wavelengths.

\section{Summary}

The present data on nearby Shapley-Ames galaxies strengthen and confirm the conclusion by Gavazzi et al. (1996) that galaxies can be regarded as an (almost) one-parameter family in parameter space. It will be challenging to reconcile the conclusion that galaxies are essentially defined by their initial mass with the presently fashionable hierarchical merging scenario. Perhaps 
this conundrum could be resolved by assuming that most of the mergers that occur in the hierarchical merger scheme are with low-mass galaxies. Such mergers would add little to the mass of the main body of the dominant galaxy, although they might make major contributions to the stellar populations in the halos of the merger products.

The Shapley-Ames galaxies within $10 \mathrm{Mpc}$ show a relatively tight correlation between luminosity and diameter. Surprisingly this correlation appears to be almost independent of a galaxy's environment that has been characterized in two different ways. On a larger scale no significant difference is found between the local luminosity-diameter relation and that observed in the Virgo cluster. Even within the Virgo cluster itself the luminositydiameter relationship appears to be essentially the same in the dense cluster core and in its lower density envelope. By the same token Gavazzi et al. (1996) find no evidence for any systematic difference between the luminosity-diameter relation in relatively nearby Abell clusters and in the lower density Great Wall region. These results are surprising because, as Gavazzi et al point out, hierarchical models of galaxy formation predict a dependence of galaxy photometric parameters on environmental conditions.

Acknowledgements. I thank Igor Karachentsev for a discussion of the nature of the power-law relationship between galaxy size and luminosity and also for information on NGC 4236. Furthermore I am also indebted to Marisa Girardi for correspondence about the distance scale used in her paper and to Thomas Puzia for his comments on a draft of this paper in which he suggested that the published magnitude of NGC 1569 might have been affected by a nearby foreground star. I am also indebted to Brent Tully and to an anonymous referee for a number of wise comments that were deeply appreciated. Thanks are also due to Brenda Parrish and Jason Shrivell for technical assistance.

\section{References}

Binggeli, B., Sandage, A., \& Tarenghi, M. 1984, AJ, 89, 64

Dalcanton, J. J., Spergel, D. N., \& Summers, F. J. 1997, ApJ, 482, 659

de Vaucouleurs, G., de Vaucouleurs, A., \& Corwin, H. 1976, Second Reference

Catalogue of Bright Galaxies (Austin: University of Texas)

Faber, S. M. 1973, ApJ, 179, 731

Faber, S. M., \& Jacson, R. E. 1976, ApJ, 204, 668

Gavazzi, G., Pierini, D., \& Boselli, A. 1996, A\&A, 312, 397

Girardi, M., Biviano, A., Giuricin, G., Mardirossian, F., \& Mezzetti, M. 1991, ApJ, 366, 393

Giuricin, G., Mardirossian, F., \& Mezzetti, M. 1988, A\&AS, 72, 151

Greggio, L., Tosi, M., Clampin, M., et al. 1998, ApJ, 504, 725

Grocholsi, A. J., et al. 2008, [arXiv:0808.0153]

Karachentsev, I. D., \& Makarov, D. L. 1999, in Galaxy Interactions at High and Low Redshifts, ed. J. Barnes, \& D. B. Sanders (Dordrechts: Kluwer), IAU Symp., 186, 109

Karachentsev, I. D., Karachentseva, V. E., Huchtmeier, W. K., \& Marakov, D. I. 2004, AJ, 127, 2031

Mo, H. J., Mao, S., \& White, S. D. M. 1998, MNRAS, 295, 319

Peebles, P. J. E. 2008, Nuovo Cimento, in press [arXiv: 0712 . 2757]

Sandage, A., \& Bedke, J. 1994, The Carnegie Atlas of Galaxies (Washington: Carnegie Institution of Washington)

Sandage, A., \& Tammann, G. A. 1981, A Revised Shapley-Ames Catalog of Bright Galaxies (Washington D. C.: Carnegie Institution)

Shapley, H., \& Ames, A. 1932, Harvard Ann., 88, 2

Tully, R. B. 1988, Nearby Galaxies Catalog (Cambridge: Cambridge University Press)

Tully, R. B., \& Fisher, J. R. 1977, A\&A, 54, 661

van den Bergh, S. 1960, Publ. David Dunlap Obs., 2, 159

van den Bergh, S. 2007, AJ, 134, 1508

Woo, J., Courteau, S., \& Dekel, A. 2008, MNRAS, in press [arXiv:0807.1331]

Zwicky, F., et al. 1961-1968, Catalogue of Galaxies and of Clusters of Galaxies (Pasadena: Caltech) 